\title{
INDICADORES DE SAÚDE - PERFIL DOS IDOSOS ASSISTIDOS NA ESTRATÉGIA SAÚDE DA FAMÍLIA DE UM MUNICÍPIO DO LESTE DE MINAS GERAIS
}

\author{
Maria Terezinha Silva Neta ${ }^{1}$; Carlos Alberto Dias ${ }^{2}$ \\ Elaine Toledo Pitanga Fernandes ${ }^{3}$; Suely Maria Rodrigues ${ }^{4}$; Marileny Boechat \\ Frauches $^{5}$ \\ ${ }^{1}$ Mestranda do Programa de Mestrado em Gestão Integrada do Território, \\ Universidade Vale do rio Doce (maria.tereza@ifmg.edu.br), Governador Valadares, \\ Minas Gerais, Brasil. \\ ${ }^{2}$ Doutorado em Psicologia Clínica. Professor Adjunto C1 da Universidade Federal \\ dos Vales do Jequitinhonha e Mucuri, Teófilo Otoni - MG \\ ${ }^{3}$ Professora doutora do Programa de Mestrado em Gestão Integrada do Território da \\ Universidade Vale do rio Doce, Governador Valadares-MG \\ ${ }^{4}$ Professora doutora do Programa de Mestrado em Gestão Integrada do Território da \\ Universidade Vale do rio Doce, Governador Valadares-MG \\ ${ }^{5}$ Professora doutora do Programa de Mestrado em Gestão Integrada do Território da \\ Universidade Vale do rio Doce, Governador Valadares-MG
}

Recebido em: 02/10/2017 - Aprovado em: 21/11/2017 - Publicado em: 05/12/2017 DOI: 10.18677/EnciBio_2017B98

\begin{abstract}
RESUMO
Nas últimas décadas, o Brasil tem passado por mudanças na estrutura etária com aumento da expectativa de vida da população idosa, e incidência de doenças crônicas. Este cenário acarretou ansiedades significativas para o governo e a sociedade e preocupações quanto ao elevado número de doenças nessa população. e utilização Este trabalho teve por objetivo identificar o perfil socioeconômico, demográfico, epidemiológico dos serviços de saúde, dos idosos assistidos pela Estratégia Saúde da Família (ESF) de um município do leste mineiro. Trata-se de um estudo descritivo, de corte transversal com abordagem quantitativa. A amostra foi composta por 163 idosos, de ambos os sexos, usuários da ESF na zona urbana de um município do leste mineiro. Foi utilizado como instrumento de coleta de dados um inventário Sociodemográfico e de Saúde. Os dados foram tabulados em planilha Excel e analisados por meio de estatística descritiva. Verificou-se que a idade média dos idosos foi de 71 anos ( $D P=8,1)$, com predomínio do sexo feminino $(71,2 \%)$, $82,8 \%$ declararam ter de 0 a 4 anos de estudo, 17,2\% moram sozinhos e recebem em média 1,3 salário mínimo ( $\mathrm{DP}=0,8)$. Observou-se que, nos últimos seis meses $85,9 \%$ realizaram pelo menos uma consulta, $89,0 \%$ informaram ter utilizado algum tipo de medicamento e um dentre quatro idosos informaram ter tido diagnóstico de pelo menos uma doença. A mudança do perfil demográfico e de saúde traz algumas preocupações e revela a necessidade de ações particulares voltadas para o idoso, para tanto é fundamental a caracterização desta população e de seus problemas de saúde.
\end{abstract}

PALAVRAS-CHAVE: perfil epidemiológico, perfil sociodemográfico, saúde do idoso. 


\title{
HEALTH INDICATORS - PROFILE OF ELDERLY PERSONS ASSISTED IN THE HEALTH STRATEGY OF THE FAMILY OF AN EASTERN MUNICIPALITY OF MINAS GERAIS
}

\begin{abstract}
In the last decades, Brazil has undergone changes in the age structure with increased life expectancy of the elderly population, and incidence of chronic diseases. This scenario has brought significant anxieties to the government and society and concerns about the high number of diseases in this population. The objective of this study was to identify the socioeconomic, demographic and epidemiological profile of the health services of the elderly assisted by the Family Health Strategy (FHS) of a municipality in eastern Minas Gerais. This is a descriptive cross-sectional study with a quantitative approach. The sample consisted of 163 elderly men and women, FHS users in the urban area of a municipality in eastern Minas Gerais. A sociodemographic and health inventory was used as a data collection instrument. The data were tabulated in an Excel spreadsheet and analyzed by means of descriptive statistics. The mean age of the elderly was 71 years $(S D=$ 8.1 ), with a predominance of females $(71.2 \%), 82.8 \%$ reported having 0 to 4 years of schooling, $17.2 \%$ live alone and receive on average 1.3 minimum wage $(S D=0.8)$. It was observed that, in the last six months, $85.9 \%$ had at least one consultation, $89.0 \%$ reported having used some type of medication and one among four elderly patients reported having been diagnosed with at least one disease. The changing demographic and health profile brings some concerns and reveals the need for particular actions geared towards the elderly, so it is fundamental to characterize this population and their health problems.
\end{abstract}

KEYWORDS: health of the elderly, epidemiological profile, sociodemographic profile

\section{INTRODUÇÃO}

Nas últimas décadas, o Brasil tem passado por profundas transformações em sua estrutura demográfica, principalmente a partir da década de 60 , fenômeno este também reconhecido em outros países em decorrência da mudança nos padrões de mortalidade e fecundidade. Este fenômeno tem ocorrido em diversos países, principalmente nos mais desenvolvidos e tem trazido inúmeras provocações sociológicas, econômicas, políticas e de saúde. Houve crescimento exponencial da população idosa em relação às demais faixas etárias (ERVANTY et al., 2015; SIMÕES, 2016).

Esse processo acelerado de envelhecimento está acarretando ansiedades significativas para o governo e a sociedade e preocupações quanto ao elevado número de doenças nessa população, incapacidades físicas e problemas nos serviços de saúde que não conseguem suprir a nova demanda. Ademais, o idoso se torna consumidor de recursos especializados, que por natureza são mais onerosos. Logo a crescente demanda por serviços de saúde atrelada ao custo destes serviços tem representado desafios à saúde pública (PIOLA et al., 2015).

Mudanças demográficas também ocasionaram alterações no perfil epidemiológico, já que as condições de vida e a medicina proporcionaram o aumento da longevidade. Isso não significa dizer que a velhice traga consigo doenças novas, apenas que as doenças mais comuns na população idosa ficam mais aparentes. Houve também, uma redução significativa de doenças infecciosas graças as mesmas condições que favoreceram a longevidade, tais como vacinação, 
melhoria da qualidade de vida, saneamento. Há de se considerar que muitas transformações físicas e psicológicas ocorrem ao longo do envelhecimento e podem trazer a diminuição da capacidade funcional e redução de atividade física que acaba por acarretar aumento da susceptibilidade para as doenças crônicas (MARCHI NETTO, 2006; ERVANTY et al., 2015).

Desta forma, o perfil epidemiológico da população idosa assistida na Estratégia Saúde da Família (ESF) afeta a condição de saúde e capacidade funcional que justifica a implementação de ações de promoção à saúde em busca da melhoria da qualidade de vida. Tais ações dependem da efetividade de políticas públicas que fortaleçam a ESF, a fim de garantir acesso, atenção integral e longitudinal a pessoa idosa para atendimento de suas necessidades (ERVANTY et al., 2015; MIRANDA et al. 2017).

Uma vez inserido na ESF, o idoso pode ter atenção integral uma vez que a equipe de saúde da família consegue estabelecer vínculo e está mais próxima da comunidade. No atendimento domiciliar esta equipe pode reconhecer aspectos culturais e familiares que interferem na saúde do idoso e na própria oferta do serviço de atenção primária a saúde (APS). De antemão há a necessidade de se conhecer a população assistida e seus problemas de saúde (RIGON et al., 2016).

Diante desta problemática, este estudo teve por objetivo identificar o perfil socioeconômico, demográfico, epidemiológico e a utilização dos serviços de saúde, dos idosos assistidos pela Estratégia Saúde da Família da zona urbana de um município do leste mineiro, que caracterizam esta parcela da população.

\section{MATERIAL E MÉTODOS}

Trata-se de um estudo transversal descritivo com abordagem quantitativa realizado com idosos cadastrados na ESF da zona urbana de um município do leste Mineiro. Foi realizado cálculo amostral, baseado no método de estimativa para proporção segundo Levy e Lemeshow (1991) que apontou uma amostra de 163 idosos incluindo $10 \%$ de possíveis perdas. Foram incluídos indivíduos de ambos os gêneros que tinham 60 anos ou mais, que estavam cadastrados na ESF, residiam na zona urbana do município e que se encontravam fisicamente independentes e com função cognitiva preservada para responder ao questionário. Para garantir a participação de idosos das 19 ESFs do município, a amostra foi distribuída conforme proporção de idosos cadastrados em cada estratégia.

Como instrumento de coleta de dados foi utilizado Inventário Sociodemográfico, baseado no estudo de Fleck (2008), que permitiu identificar o perfil sociodemográfico, os indicadores de saúde e a utilização dos serviços de saúde. As variáveis independentes incluídas foram: idade, sexo, situação conjugal, escolaridade, renda mensal individual, arranjo familiar, número de consultas médicas realizadas na ESF (nos últimos 6 meses), número de medicamentos utilizados diariamente (nos últimos 6 meses) e número de doenças diagnosticadas pelo médico do SUS.

O local de residência, bem como a identificação dos idosos que preenchiam aos critérios de inclusão, foi levantado por meio de análise dos prontuários com auxílio dos profissionais de saúde que compunham a ESF sem interferir na rotina de trabalho. Foi realizado um estudo piloto com 10 idosos que também preenchiam os critérios de inclusão em uma Clínica Odontogeriátrica do curso de Odontologia de uma Universidade do leste de Minas, a fim de averiguar a abordagem das questões, bem como o tempo necessário para a aplicação do questionário. 
Após o registro dos endereços, os idosos foram orientados quanto ao objetivo do estudo e convidados a participar da pesquisa. Foram ainda orientados quanto a participação voluntária, sem ônus e que a participação poderia ser encerrada a qualquer momento. Após a assinatura do Termo de Consentimento Livre e Esclarecido (TCLE) o questionário foi aplicado em domicílio utilizando-se de entrevistas realizadas pelos pesquisadores, nos períodos de maio e junho de 2014 e março a julho de 2015. Esta pesquisa foi aprovada pelo Comitê de Ética em Pesquisa da Universidade Vale do Rio Doce (UNIVALE), com o protocolo CEP/UNIVALE 532.075.

Os dados foram tabulados em uma planilha do Microsoft Office Excel 97-2003 e agrupados, levando-se em consideração as variáveis incluídas no Inventário Sóciodemográfico. Sumariamente procedeu-se a análise estatística descritiva dos dados em que os achados foram discutidos conforme revisão literária prévia. Para testar a hipótese de que a faixa etária, renda e a escolaridade se correlacionavam com o número de consultas e doenças foi aplicado teste de correção de Pearson's Chi-squared por meio do software R, versão 3.2.2, livremente disponível no site https://www.r-project.org. Foi adotando um intervalo de confiança de $95 \%$ em que, para associação positiva entre as varáveis 0 valor encontrado para $p$ deveria ser inferior a $0,05(p<0,05)$.

\section{RESULTADOS E DISCUSSÃO}

\section{Perfil socioeconômico e demográfico dos idosos participantes da pesquisa}

Participaram da pesquisa 163 idosos usuários da ESF da zona urbana do município do leste mineiro. Obedecendo a um nível de confiança de 99\%, a amostra demostrou significativa representatividade da subpopulação de indivíduos idosos do referido município, uma vez que é estatisticamente semelhante a população de acordo com o último censo demográfico pelo Instituto Brasileiro de Geografia Estatística (IBGE). A tabela 1 abaixo confirma tal inferência, uma vez que apresenta a distribuição da faixa etária dos idosos que compuseram a amostra, bem como a distribuição entre as faixas etárias e a frequência esperada segundo IBGE (2010).

TABELA 1 - Distribuição da faixa etária dos idosos da amostra em relação à proporção apresentada pelo IBGE em 2010 para a população deste estudo.

\begin{tabular}{lcccc}
\hline Faixa Etária* & $\begin{array}{c}\text { Frequência } \\
\text { (pesquisa) } \\
\text { Mulheres }\end{array}$ & $\begin{array}{c}\text { Observada } \\
\text { Homens }\end{array}$ & $\begin{array}{c}\text { Frequência Esperada (IBGE) } \\
\text { Mulheres }\end{array}$ & Homens \\
\hline De 60 a 64 anos & 26 & 10 & 35 & 14 \\
De 65 a 69 anos & 32 & 13 & 28 & 11 \\
De 70 a 74 anos & 19 & 9 & 22 & 9 \\
De 75 a 79 anos & 21 & 5 & 16 & 6 \\
De 80 a 84 anos & 16 & 5 & 10 & 3 \\
De 85 a 89 anos & 2 & 2 & 5 & 2 \\
Mais de 90 anos & 2 & 1 & 2 & 1 \\
Total Geral & 118 & 45 & 118 & 45 \\
\hline
\end{tabular}

${ }^{*} p=0,03903$

Fonte: Adaptado de Brasil (2010) e dados da pesquisa.

Ainda segundo a faixa etária, constatou-se com $95 \%$ de confiança, que a idade média da amostra foi de 71,3 anos $(\mathrm{DP}=8,1)$, variado de 60 anos até 102 anos. Segundo dados do IBGE (2010), no município de estudo, $53,7 \%$ da população idosa possuía menos de 70 anos em 2010, já na amostra 50,3\% apresentou menos de 70 anos $(Q 25 \%=65)$, seguidos de $31,9 \%$ com 71 a 80 anos $(Q 75 \%=77,8)$, ENCICLOPÉDIA BIOSFERA, Centro Científico Conhecer - Goiânia, v.14 n.26; p.1154 2017 
conforme apresentado na tabela 2. Para o Brasil, há a expectativa que em 2030, $56,5 \%$ da população idosa tenha menos de 70 anos. Também, em estudos com população idosa inserida nos serviços de saúde pública, Araújo et al. (2014) e Barbosa et al. (2015) encontraram respectivamente $43,0 \%$ e $45,9 \%$ da população idosa estudada dentro desta faixa etária.

TABELA 2 - Características socioeconômicas e demográficas dos idosos pesquisados em um município do leste mineiro.

\begin{tabular}{|c|c|c|}
\hline Variáveis & Frequência & $\%$ \\
\hline \multicolumn{3}{|l|}{ Idade } \\
\hline Menos de 70 & 82 & 50,3 \\
\hline De 70 a 79 & 52 & 31,9 \\
\hline De 80 a 89 & 26 & 16,0 \\
\hline 90 e mais & 03 & 1,8 \\
\hline \multicolumn{3}{|l|}{ Sexo } \\
\hline Masculino & 47 & 28,8 \\
\hline Feminino & 116 & 71,2 \\
\hline Estado civil & & 41,7 \\
\hline Casado (a) & 68 & 11,0 \\
\hline Solteiro (a) & 18 & 36,2 \\
\hline Viúvo (a) & 59 & 2,5 \\
\hline Em união consensual & 04 & 8,6 \\
\hline Separado (a) & 14 & \\
\hline \multicolumn{3}{|l|}{ Escolaridade (anos) } \\
\hline 0 & 53 & 32,5 \\
\hline De 1 a 4 & 82 & 50,3 \\
\hline De 5 a 8 & 13 & 8,0 \\
\hline De 9 a 13 & 15 & 9,2 \\
\hline \multicolumn{3}{|l|}{ Situação de Trabalho } \\
\hline Empregado & 9 & 5,5 \\
\hline Desempregado & 6 & 3,7 \\
\hline Auxílio-doença & 1 & 0,6 \\
\hline Aposentado & 88 & 54,0 \\
\hline Aposentado e trabalha & 9 & 5,5 \\
\hline Do lar & 16 & 9,8 \\
\hline Pensionista & 33 & 20,2 \\
\hline Outro Auxílio & 1 & 0,6 \\
\hline \multicolumn{3}{|l|}{ Com quem mora } \\
\hline Esposo (a) & 52 & 31,9 \\
\hline Filhos & 50 & 30,7 \\
\hline Netos & 5 & 3,1 \\
\hline Bisnetos & 3 & 1,8 \\
\hline Irmão & 9 & 5,5 \\
\hline Amigos & 0 & 0,0 \\
\hline Sozinho & 28 & 17,2 \\
\hline Esposa + filhos & 16 & 9,8 \\
\hline \multicolumn{3}{|l|}{ Renda familiar } \\
\hline Menos de 1 salário mínimo & 3 & $1,84 \%$ \\
\hline De 1 a 2 salários mínimos & 141 & $86,50 \%$ \\
\hline 3 ou mais salários mínimos & 9 & $5,52 \%$ \\
\hline Não responderam & 10 & $6,13 \%$ \\
\hline Total & 163 & $100 \%$ \\
\hline
\end{tabular}

Fonte: Dados da pesquisa. 
No Brasil, as pesquisas demográficas apontam que $55,1 \%$ da população idosa, pertencem à faixa etária de 60 a 69 anos. Tal fenômeno se justifica pelas altas taxas de natalidade entre os anos 1950 a 1960, conhecido como baby boomers. Ou seja, neste período, também conhecido pela explosão demográfica, houve crescimento acentuado no número da natalidade e diminuição da mortalidade. Atualmente os babies boomers (indivíduos nascidos entre os anos 1950 a 1960), graças a melhoria da qualidade de vida e redução da mortalidade chegaram na faixa etária de 60 a 69 anos e ganham o título de elderly boomers (BURLÁ et al., 2013; CAMARANO, 2014).

Quanto ao gênero, conforme apresentado na tabela 2, $116(71,2 \%)$ são do gênero feminino e $47(28,8 \%)$ do gênero masculino. Já no município pesquisado, a população idosa feminina corresponde a $57,6 \%$ da população idosa e $47,4 \%$ do gênero masculino segundo IBGE (2010). Pilger et al. (2011) e Araújo et al. (2014) também encontraram respectivamente $64,4 \%$ e $64,0 \%$ da população idosa do gênero feminino.

Há de se esperar uma população idosa com maior prevalência de mulheres uma vez que os valores observados para a expectativa média de vida em 2010 foram de 77,7 anos para mulheres e 70,1 para homens com expectativa de crescimento para 86,1 anos e 82,6 respectivamente em 2050 . Se forem reduzidos significativamente ou eliminados os óbitos por causas externas, que reduziu 3,8 anos da expectativa de vida masculina em 2012, há a hipótese de aumento da expectativa masculina comparada a feminina. Mas ainda será observada maior predominância do gênero feminino (CAMARANO, 2014).

Tal fenômeno tem sido reconhecido como 'feminilização da velhice', a mulher tem vivido mais tempo comparado ao homem nas diversas faixas etárias. Fenômeno que também ocorre no município estudado, conforme apresentado na figura 1 , no Brasil e também em diversos países. No Brasil, em 2000 haviam 81 homens idosos para cada 100 mulheres idosas. Estima-se que, em 2050 este número cairá para 76 a cada 100 respectivamente e ainda nas faixas etárias acima de 80 anos serão 25 para 100 respectivamente (ERVANTY et al., 2015).

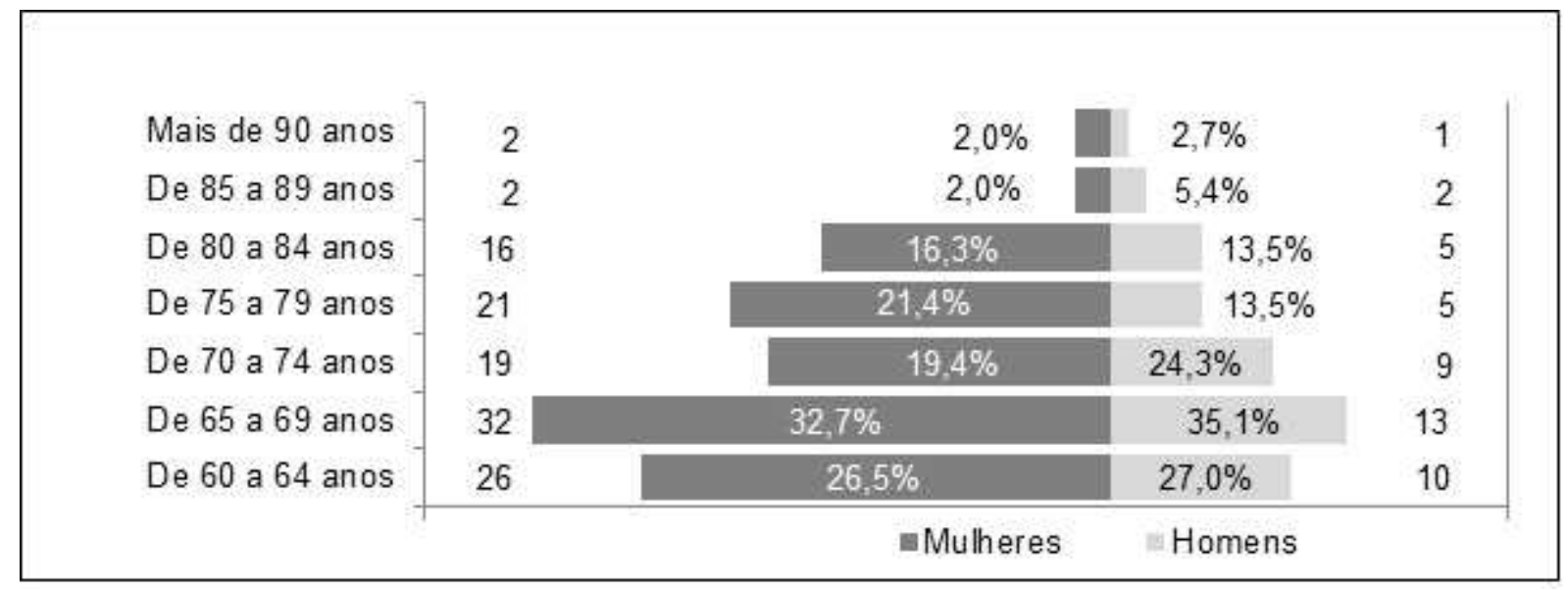

FIGURA 1: Distribuição da amostra segundo gênero e Faixa etária Fonte: dados da pesquisa

Em um estudo com mulheres idosas, Merighi et al. (2013) identificaram que a mulher ao envelhecer almeja manter-se fisicamente e socialmente ativa mesmo com a presença de limitações e alterações fisiológicas interpostas pela idade. Para essas 
mulheres há a necessidade na manutenção da capacidade funcional e promoção da saúde a fim de evitar e retardar a surgimento de doenças incapacitantes.

Mesmo que em todos os países do mundo, nasçam mais homens que mulheres, neste grupo as taxas de mortalidade são maiores em todas as faixas etárias. Os indivíduos do sexo masculino estão mais expostos a violência urbana, no trânsito e no trabalho, maior uso e/ou abuso de drogas, álcool e tabaco. As mulheres ainda têm o fato de proteção hormonal atribuído ao estrogênio e maior preocupação com a saúde e autocuidado o que a faz buscar mais pelos serviços de saúde. Tais fatores contribuem para que, com o aumento das faixas etárias, seja observado maior proporção de mulheres (SANTOS ; CUNHA, 2014; IBGE, 2016).

Quanto ao estado civil, 42,8\% dos pesquisados declararam serem casados ou viverem em união consensual e 55,8\% não possuem cônjuges por serem solteiros, viúvos ou separados. Areosa e Virginia (2012), encontraram 49,0\% de idosos solteiros, viúvos e casados. Já Barbosa et al. (2015) encontraram 60,1\% de indivíduos na mesma condição, ao estudarem idosos assistidos nos serviços de saúde pública. Esta condição também pode representar impactos a saúde uma vez que a solidão e principalmente o processo de luto e viuvez são fatores estressores que se associam à diminuição do autocuidado, contribuem para o aumento da exposição à riscos ambientais e comportamento inseguro (GOMES et al., 2013).

Em relação ao nível de escolaridade, $82,8 \%$ declararam ter de zero a quatro anos de estudo, do total $32,5 \%$ informaram ser analfabetos. A média de anos de estudo apresentada pelos idosos da pesquisa foi de 2,9 anos ( $D P=3,2)$. Apenas $9,2 \%$ podem ter concluído o ensino fundamental ou ensino médio regular já que tem de nove a 13 anos de estudo. O trabalho desenvolvido por Barbosa et al. (2015) também demonstrou resultados semelhantes. Houve, ao longo dos anos, queda significativa nas taxas de analfabetismo nas diversas faixas etárias, mas a baixa escolaridade e analfabetismo ainda é muito prevalente na população acima de 65 anos. Cerca de $25,7 \%$ da população acima desta faixa etária não sabe ler ou escrever e em 2015, 60,5\% de pessoas analfabetas tinham idade acima de 55 anos, o que revela mais um aspecto de vulnerabilidade à saúde do idoso (IBGE, 2016).

Este cenário traz preocupação à gestão de saúde pública uma vez que o grau de escolaridade se associa ao perfil epidemiológico de uma população e à distribuição dos indicadores de saúde. Os baixos índices de escolaridade podem trazer ao idoso dificuldades no autocuidado, administração de medicamentos além da interpretação de orientações escritas como as receitas médicas, bulas e dietas. Já maiores níveis de escolaridade podem vir acompanhados de melhor percepção de saúde, busca pelos serviços de saúde e adesão às práticas de vida saudável (GARCIA ; FACCHINI, 2009).

Apesar das quedas nas taxas de analfabetismo no país, a alfabetização de adultos e especialmente idosos é um desafio para o governo e tem contribuído para a distribuição desigual de indicadores de saúde. Também se relaciona com entrada no mercado de trabalho, acesso aos serviços de saúde, tratamento e recuperação. Além desta problemática desafiar os profissionais de saúde, principalmente os que trabalham na APS, na condução do cuidado ao idoso e manutenção da comunicação. Será necessário ainda que estes profissionais compreendam as territorialidades, as ações e práticas do idoso no seu cotidiano a partir do território que permitirá a identificação de aspectos que trazem riscos à saúde e vulnerabilidade (MONKEN ; BARCELLOS, 2005; GARCIA ; FACCHINI, 2009).

Quanto a renda, $54,0 \%$ se declaram aposentados, e $20,20 \%$ pensionistas. A população idosa da pesquisa tem renda média de 1,3 salário mínimo $(\mathrm{DP}=0,8)$ 
variando de zero a seis salários, e $86,50 \%$ destes vivem com um a dois salários mínimos e 5,52\%\% recebe três ou mais salários mínimos. Assim como a amostra, no Brasil grande parcela da renda dos idosos não advém do trabalho e sim de aposentadorias e outros fundos assistenciais, que são necessários para a subsistência desta população. No país em 2015, 75,6\% dos idosos eram aposentados e/ou pensionistas e ainda $69 \%$ destes tinham estas como única fonte de rendimento, também fator de vulnerabilidade (IBGE, 2016).

Assim como baixos índices de escolaridade, a baixa renda também se relaciona a piores condições de saúde e diminuição da capacidade funcional uma vez que o idoso pode ter pior padrão de vida, alimentação inadequada e dificuldades para o acesso aos serviços de saúde. A de se considerar ainda que a moradia também envolve recursos, desta forma os bairros podem ter aspectos distintos, como infraestura, saneamento e até mesmo aspectos culturais de acordo com a renda de seus moradores (MONKEN ; BARCELLOS, 2005; NERI ; SOARES, 2007).

Em um estudo com idosos, Reis et al. (2015) identificaram que a renda, estado civil e faixa etária estão relacionadas à diminuição da capacidade funcional. Com a perda ou diminuição da renda com a idade ou aposentadoria, os fatores socioeconômicos trazem impactos a saúde e manutenção da qualidade de vida que pode lhe conferir impactos negativos sobre a saúde.

Quanto ao arranjo familiar $72,8 \%$ dos idosos vivem com o cônjuge, filhos ou cônjuge e filhos. Pilger et al. (2011) encontraram 86,4\% de idosos na mesma condição. Entretanto Machado e Vieira (2015), identificaram que apenas $41,9 \%$ dos idosos de seu estudo moram acompanhados. Todavia, apesar de uma formação ainda tradicional, o arranjo familiar vem sofrendo alterações graças às mudanças no perfil demográfico. Tem sido registrado o aumento dos arranjos domiciliares com uma única pessoa, destacando mulheres com 50 anos ou mais (IBGE, 2016).

Araújo et al. (2013) em um estudo que avaliou o perfil de cuidadores de idosos identificou que a maioria dos idosos era cuidada por cônjuges e filhos sem a presença de um cuidador externo remunerado. Apesar de muitos relatarem exaustão e falta de conhecimento necessário para a função, exerciam papel fundamental na prestação de cuidados básicos como alimentação, higiene e medicação. Destaca-se a importância da ESF na orientação das famílias e suporte ao cuidado do idoso a fim de suprir esta necessidade.

Tais características sociodemográficas apresentadas pela população estudada demostram particularidades que podem auxiliar no reconhecimento de suas vivências e cotidiano, processos de territorialização, as necessidades e problemas de saúde. Já que cada território tem sua população, além de reconhecer os limites geográficos de abrangência de uma equipe de saúde da família, o trabalho precisa ser pautado nos contextos territoriais da população de abrangência, para que as práticas sejam apropriadas e próximas ao público a que se destinam. É necessário aproximar o trabalho dessas equipes aos problemas de saúde e onde acontecem a fim de resolvê-los ou amenizá-los (MONKEN ; BARCELLOS, 2005).

As equipes de saúde da família, ao promoverem ações de vigilância em saúde precisam reconhecer além de características de ocupação do lugar como núcleos habitacionais, sistema de água e saneamento e condições ecológicas, consigam relacionar trabalho, renda, escolaridade, cultura à vida cotidiana, a identidade e territorialidade. Uma população ao utilizar recursos básicos pode valerse de métodos que afetam a saúde que merecem ser estudados com o objetivo de identificar situações de vulnerabilidade (MONKEN ; BARCELLOS, 2005). 
A ESF precisa pensar no território de atuação além de seu caráter político, administrativo em que há diversas vezes disputas entre poderes institucionalizados. Deve se pensar na categoria território como multidimensional em que tanto a gestão de saúde quando a população assistida vivencia o território e as relações que neste se estabelecem (MONKEN ; BARCELLOS, 2005).

Monken et al. (2008) acrescentam ainda que no território da saúde coletiva, onde atuam essencialmente as ESF, devem ser desenvolvidas ações preventivas voltadas às características demográficas e epidemiológicas permitindo espaço de troca que visem melhoria contínua da qualidade de vida. Desta forma, estudos de diagnóstico e caracterização da população servem de subsídio para a gestão de saúde pública no planejamento de ações.

\section{Indicadores de saúde e utilização dos serviços de saúde}

Sistemas de informações em saúde (SIS) têm demonstrado como ferramentas necessárias para a gestão e saúde e para a tomada de decisão, já reconhecida pela Organização Mundial da Saúde (OMS) como um dos pilares dos Sistemas de Saúde. Para tanto é fundamental que os SIS, sejam planejados e estruturados de forma a permitir a geração de informação acerca de determinantes de saúde, estado de saúde de uma população e ainda sobre o próprio desempenho que fomentem a orientação das práticas em saúde e utilização de recursos (MUTALE et al., 2013; LIMA et al., 2015).

Diversas metodologias de coleta de dados podem ser aplicadas para obtenção de informação como a utilização de censo, notificação compulsória de agravos, pesquisas em domicilio, que com tratamento estático permitem a identificação pontual de problemas, instalação de rotinas, planejamento e monitoramento da saúde. Mas, as deficiências de um SIS, como a duplicação de informação, baixa capacitação profissional ou incapacidade de análise dos dados podem mascarar problemas e atrasar a oferta de APS. Em um estudo com gestores, Lima et al. (2015) identificaram baixa utilização e conhecimento dos SIS e que as ações em saúde são planejadas de acordo com a livre demanda dos usuários o que retarda as intervenções em saúde pública (MUTALE et al., 2013; LIMA et al., 2015).

Compreendendo a necessidade da informação em saúde, identificou-se neste estudo que $14,1 \%$ da população idosa pesquisada não realizou nenhuma consulta nos últimos seis meses que antecederam a coleta de dados, 59,5\% realizam de uma a três consultas e $26,4 \%$ realizam quatro ou mais consultas conforme apresentado na tabela 3. Já segundo o Sistema de Informação Básica (SIAB, 2017), foram realizadas em 2014, período de início da coleta de dados 37.834 consultas apenas pela população com 60 anos ou mais. Ou seja, cada idoso cadastrado na ESF, fez em média 2,8 consultas no ano de 2014.

TABELA 3 - Indicadores de saúde dos idosos pesquisados em um município do leste mineiro no período de 2014-2015.

\begin{tabular}{ccc}
\hline Variáveis & Freq. & $\%$ \\
\hline № de Consultas Médicas & 23 & $14,1 \%$ \\
Nenhuma & 97 & $59,5 \%$ \\
1 a 3 & 43 & $26,4 \%$ \\
4 ou mais & 18 & $11,0 \%$ \\
№ de Medicamentos & 74 & $45,4 \%$ \\
Nenhum & &
\end{tabular}




\begin{tabular}{ccc}
\hline Variáveis & Freq. & $\%$ \\
\hline 4 ou mais & 71 & $43,6 \%$ \\
No de doenças & & \\
Nenhuma & 30 & $18,4 \%$ \\
1 & 63 & $38,7 \%$ \\
2 & 39 & $23,9 \%$ \\
3 & 17 & $10,4 \%$ \\
4 ou mais & 14 & $8,6 \%$ \\
\hline Total & 163 & $100 \%$
\end{tabular}

Fonte: Dados da pesquisa.

Silva et al. (2017) encontraram associação entre o maior número de consultas, limitação funcional e a ocorrência de internação. Apesar de reconhecer como positiva a procura pela consulta médica por ser uma medida prevencionista atribuíram as altas médias à baixa resolubilidade dos problemas de saúde que poderiam ser evitados com ações de APS. Também considera o envelhecimento populacional atrelado ao aumento da demanda e necessidade de ampliação da oferta de serviços de APS, ao aumento de idosos com doenças crônicas e limitação funcional.

O teste de correlação de Pearson nesta pesquisa, encontrou um valor $p$ de 0,6051 , ou $60,51 \%$ ao associar a renda ao número de consultas e um valor $p$ de 0.3992 ou $39,92 \%$ ao associar escolaridade também ao número de consultas médicas. Adotando-se um nível de significância (a) de $5 \%$, em que o valor encontrado para $p$ é maior que este valor $0,05(p>0,05)$ rejeita-se a hipótese de que a escolaridade ou a renda se correlacione ao número de consultas médicas realizadas pela população idosa estudada.

Em relação ao número de medicamentos $89,0 \%$ dos idosos relatam fazer uso de pelo menos um medicamento. $43,6 \%$ utilizam quatro ou mais medicamentos. Tavares et al. (2015) afirmam que a utilização de medicamentos no Brasil, para o tratamento de doenças crônicas é elevado com destaque para os anti-hipertensivos, medicamentos para tratamento de diabetes e asma com aumento significativo de $80 \%$ na população geral. Observou-se também maior utilização de anti-hipertensivos no gênero feminino nas maiores faixas etárias, que pode estar relacionada a maior utilização dos serviços de saúde.

É importante destacar que o número de idosos que não fizeram nenhuma consulta nos últimos seis meses que antecederam a coleta de dados $(14,1 \%)$ é maior que o número de idosos que não utilizam nenhum medicamento $(11,0 \%)$. Cassoni et al. (2014) em um estudo no município de São Paulo, identificaram que $25,0 \%$ dos idosos utilizam medicamentos inapropriados que acabam por se associar à sua internação e aumento da mortalidade. Identificaram ainda que o diagnóstico de duas ou mais doenças e a utilização de cinco ou mais medicamentos se associou a utilização indevida de medicamentos. Já Silva et al. (2010) identificaram que $25,0 \%$ dos idosos relatam ter dificuldades na utilização de medicamentos o que reforça a necessidade de orientação e educação para a saúde.

Neves et al. (2013) associaram a utilização de diferentes fármacos a menor escolaridade, a presença de duas ou mais doenças e ao número de consultas, fenômenos que também ocorreram nesta pesquisa. Quanto ao número de doenças $18,4 \%$ dos idosos afirmaram não terem nenhuma doença. O que chama atenção é o elevado número de idosos, $42,9 \%$, que relataram ser portadores de duas ou mais doenças. Quatro ou mais doenças é mais incidente entre as mulheres, 22,0\% contra 
13,3\% dos homens. Merighi et al. (2013) atribuíram esta ocorrência a maior preocupação da mulher com a saúde e sua procura pelos serviços de saúde que culminam em mais diagnósticos em mulheres em relação aos homens.

Pereira et al. (2015) encontraram resultados semelhantes e ainda identificaram que a hipertensão arterial é a doença mais prevalente entre os idosos seguidos de diabetes, osteoporose, ansiedade e doenças cardiovasculares. Neves et al. (2013) também identificaram a hipertensão arterial como a doença mais prevalente na população idosa. Neste estudo, o teste de correlação de Pearson, encontrou um valor $p$ de 0.748 , ou $74,80 \%$ ao associar a faixa etária ao número de doenças, um valor $p$ de 0.3065 ou $30,65 \%$ ao associar renda ao número de doenças e por fim um valor $p$ de 0,6674 ou $66,74 \%$ ao associar escolaridade também ao número de doenças. Adotando-se um nível de significância (a) de $5 \%$, em que o valor encontrado para $p$ é maior que $0,05(p>0,05)$ rejeita-se a hipótese de que a faixa etária, a escolaridade ou a renda se correlacione ao número de doenças diagnosticas na população idosa.

A partir do estudo das características epidemiológicas levantadas nesta pesquisa é possível inferir que a população de estudo demonstra um perfil de vulnerabilidade que também se repetiu em outras regiões do país. Tal cenário confirma a necessidade de promoção da saúde e envelhecimento saudável para prevenir o acometimento de doenças crônicas. Ressalta-se ainda a importância do estabelecimento de vínculo entre a esquipe e o idoso que facilite a orientação continua quanto a alimentação saudável, comportamento de risco, terapêutica e o incentivo quanto ao envelhecimento ativo (RIGON et al., 2016).

Tais levantamentos são necessários para diagnóstico das condições de vida de uma população que fundamentam as práticas em saúde. Apesar de a doença manifestar-se em um indivíduo, as condições de saúde de uma população se revelam em um lugar cheio de história, cultura, perfil sociodemográfico, disponibilidade de serviços de saúde e ainda condições físicas e ambientais que associados contribuem para o surgimento de agravos a saúde (MONKEN ; BARCELLOS, 2005).

\section{CONCLUSÃO}

Os resultados deste estudo permitiram identificar na análise do perfil dos idosos assistidos na ESF, características de vulnerabilidade social e epidemiológica que podem estar associadas à ocorrência de agravos a saúde. Os achados deste estudo, que também foram descritos em outras pesquisas nas diversas regiões do país, podem contribuir com a gestão de saúde na análise e aplicação de práticas em saúde voltadas às características desta população de modo a atender suas necessidades, resolver ou amenizar seus problemas de saúde. Considerando a transição demográfica e epidemiológica vivida no país, há a necessidade de se entender suas consequências e adequar os sistemas, políticas e profissionais a esta realidade, para garantir a assistência integral ao idoso.

\section{REFERÊNCIAS}

ARAUJO, J. S.; VIDAL, M. G.; BRITO, F. N.; GONÇALVES, D. C. A.; et al. Perfil dos cuidadores e as dificuldades enfrentadas no cuidado ao idoso, em Ananindeua, PA. Revista Brasileira de Geriatria e Gerontologia, v. 16, n. 1, p. 149-158, fev-mar, 2013. Disponível em: <http://dx.doi.org/10.1590/S1809-98232013000100015>. DOI: 10.1590/S1809-98232013000100015 
ARAÚJO, L. U. A.; GAMA, Z. A. S.; NASCIMENTO, F. L. A.; DE OLIVEIRA, H. F. V.; AZEVEDO. W. M.; et al. Avaliação da qualidade da atenção primária à saúde sob a perspectiva do idoso. Ciência \& Saúde Coletiva, v. 8, n. 19, p. 3521-3532, 2014. Disponível em: <http://dx.doi.org/10.1590/1413-81232014198.21862013.>. DOI: 10.1590/1413-81232014198.21862013

AREOSA, C.; VIRGINIA. S. Relações sociais e familiares do idoso atendido pela UNISC. Revista Brasileira de Ciências do Envelhecimento Humano, v. 7, n. supl. 1, p. 124-134, 2012. Disponível em: <http://dx.doi.org/10.5335/rbceh.2012.1118>. DOI: http://dx.doi.org/10.5335/rbceh.2012.1118

BARBOSA, T. F. K.; OLIVEIRA, R. L. M.; FERNANDES, F. G. M. Caracterização sociodemográfica e clínica de idosos atendidos na atenção primária de saúde. Journal of Nursing UFPE/Revista de Enfermagem UFPE, v. 9, n. 10, p. 94929499, 2015. Disponível em:<http://dx.doi.org/10.5205/reuol.7944-69460-1SM.0910201510>. DOI: 10.5205/reuol.7944-69460-1-SM.0910201510

BRASIL. Conselho Nacional de Secretários de Saúde. Atenção Primária e Promoção da Saúde. - Brasília: CONASS, 2007. 232 p.

BURLÁ, C.; CAMARANO, A. A.; KANSO, S.; FERNANDES, D.; NUNES, R. Panorama prospectivo das demências no Brasil: um enfoque demográfico. Ciência \& Saúde Coletiva, n. 18, v. 10, p. 2949-2956, 2013. Disponível em: <http://dx.doi.org/10.1590/S1413-81232013001000019>. DOI: 10.1590/S141381232013001000019

CAMARANO, A. A. Perspectivas de crescimento da população brasileira e algumas implicações. In: Novo Regime Demográfico uma nova relação entre população e desenvolvimento? Rio de Janeiro: Ipea, 2014. 658 p.

CASSONI, T. C. J.; CORONA, L. P.; ROMANO-LIEBER, N. S.; SECOLI, S. R.; DUARTE, Y. A. O. et al. Uso de medicamentos potencialmente inapropriados por idosos do Município de São Paulo, Brasil: Estudo SABE. Caderno de Saúde Pública, Rio de Janeiro, v. 8, n. 30, p. 1708-1720, ago/2014. Disponível em: <http://dx.doi.org/10.1590/0102-311X00055613>. DOI: 10.1590/0102-311X00055613

ERVANTY, L. R.; BORGES, G. M.; JARDIM, A. P. Mudanças demográficas no Brasil no início do Século XXI: Subsídios para a projeção da população. Rio de Janeiro: IBGE, Coordenação de População e Indicadores Sociais, 2015. 156 p.

FLECK, M. P. A. A Avaliação de Qualidade de Vida: guia para profissionais da saúde. Porto Alegre: Artemed, 2008. 228 p.

GARCIA, L. P e FACCHINI, L. A. Evolução e avanços da saúde da família e os 20 anos do Sistema único de Saúde no Brasil. In: Brasil. Ministério da saúde. Secretaria de vigilância em Saúde. Departamento de análise de Situação de Saúde. Brasil 2008: 20 anos de Sistema único de Saúde. - Brasília: Ministério da saúde: 2009. $416 \mathrm{p}$. 
GOMES, M. M. F.; TURRA, C. M.; FIGOLI, M. G. B.; DUARTE, Y. A. O.; LEBRÃO, M. L. Associação entre mortalidade e estado marital: uma análise para idosos residentes no Município de São Paulo, Brasil, Estudo SABE, 2000 e 2006. Caderno de Saúde Pública, Rio de Janeiro, v. 29, n. 3, p. 566-578, mar, 2013. Disponível em: <http://dx.doi.org/10.1590/S0102-311X2013000300014>. DOI: 10.1590/S0102$311 \times 2013000300014$

IBGE - Instituto Brasileiro de Geografia e Estatística. Censo 2010. Disponível em: http://cidades.ibge.gov.br/painel/populacao.php?codmun=312770

IBGE - Instituto Brasileiro de Geografia e Estatística. Síntese de Indicadores Sociais: uma análise das condições de vida da população brasileira. Rio de Janeiro; 2016. 141 p.

LEVY, P. S.; LEMESHOW, S. Sampling of populations - methods and applications. 2. ed. New York: John Wiley and Sons,1991. 420 p.

LIMA, K. W. S.; ANTUNES, J. L. F.; SILVA, Z. P. Percepção dos gestores sobre o uso de indicadores nos serviços de saúde. Saúde Sociedade. São Paulo, v. 24, n. 1 , p. 61-71, 2015. Disponível em: <http://dx.doi.org/10.1590/S010412902015000100005>. DOI 10.1590/S0104-12902015000100005

MACHADO, A.; VIEIRA, M. C. U. Impacto de fatores socioeconômicos na funcionalidade da pessoa idosa portadora de condições crônicas. Revista de Enfermagem da UFSM, v. 5, n. 1, p. 81-91. 2015. Disponível em: <http://dx.doi.org/10.5902/2179769213703>. DOI: 10.5902/2179769213703

MARCHI NETTO, F. L. Aspectos biológicos e fisiológicos do envelhecimento humano e suas implicações na saúde do idoso. Pensar a Prática, v. 7, n. 1, p. 7584, nov/2006. Disponível em: <https://doi.org/10.5216/rpp.v7i1.67>. DOI: 10.5216/rpp.v7i1.67

MERIGHI, M. A. B.; OLIVEIRA, D. M.; JESUS, M. C. P.; SOUTO, R. Q.; THAMADA, A. A. Mulheres idosas: desvelando suas vivências e necessidades de cuidado. Revista Escola Enfermagem, n. 47, v. 2, p. 408-414, 2013. Disponível em: <http://dx.doi.org/10.1590/S0080-62342013000200019>. DOI: $10.1590 / S 0080-$ 62342013000200019

MIRANDA, G. M. D; MENDES, A. C. G; SILVA, A. L. A. Desafios das políticas públicas no cenário de transição demográfica e mudanças sociais no Brasil. Interface, Botucatu, v. 21, n. 61, p. 309-320, 2017. Disponível em: <http://dx.doi.org/10.1590/1807-57622016.0136> DOI: 10.1590/1807-57622016.0136

MONKEN, M.; PEITER, P.; BARCELLOS, C.; ROJAS, L. I.; NAVARRO, M. et al. O território da Saúde: construindo referencias para a análise da saúde em saúde e ambiente, $2008 . \quad 20 \quad$ p. $\quad$ Disponível em: <http://www.epsjv.fiocruz.br/upload/ArtCient/21.pdf>. Acesso em: 22 nov. 2016.

MONKEN, M.; BARCELLOS, C. Vigilância em saúde e território utilizado: possibilidades teóricas e metodológicas. Caderno de Saúde Pública, Rio de 
Janeiro, v. 21, n. 3, p. 898-906, mai/jun, 2005. Disponível em: <http://dx.doi.org/10.1590/S0102-311X2005000300024>. DOI: $10.1590 / S 0102-$ 311 X2005000300024

MUTALE, W.; CHINTU, N.; AMOROSO, C.; AWOONOR-WILLIANS, K.; PHILLIPS, $\mathrm{J}$; et al. Improving health information systems for decision making across five subSaharan African countries: implementation strategies from the African Health Initiative. BioMed Central Health Services Research, London, v. 13, p. S9, 2013. Supplement 2. Disponível em: <https://doi.org/10.1186/1472-6963-13-S2-S9>. DOI: 10.1186/1472-6963-13-S2-S9

NERI, M. C; SOARES, W. L. Estimando o impacto da renda na saúde através de programas de transferência de renda aos idosos de baixa renda no Brasil. Caderno de Saúde Pública, Rio de Janeiro, n. 23, v. 8, p. 1845-1856, ago/2007. Disponível em:< http://dx.doi.org/10.1590/S0102-311X2007000800011.>. DOI: 10.1590/S0102311 X2007000800011.

NEVES, S. J. F.; MARQUES, A. P. O.; LEAL, M. C. C.; DINIZ, A. S.; MEDEIROS, T. S.; et al. Epidemiologia do uso de medicamentos entre idosos em área urbana do Nordeste do Brasil. Revista de Saúde Pública, v. 4, n. 47, p. 759-768, 2013. DOI: <http://dx.doi.org/10.1590/rsp.v47i4.76608>. $\quad$ DOI: 10.1590/S00348910.2013047003768 .

PEREIRA, D. S.; NOGUEIRA, J. A. D.; SILVA, C. A. B. Qualidade de vida e situação de saúde de idosos: um estudo de base populacional no Sertão Central do Ceará. Revista Brasileira de Geriatria e Gerontologia, Rio de Janeiro, v. 4, n. 18, p. 893908, 2015. Disponível em: <http://dx.doi.org/10.1590/1809-9823.2015.14123>. DOl: 10.1590/1809-9823.2015.14123.

PILGER, C. MENON, M. H. MATHIAS, T. A. F. Características sociodemográficas e de saúde de idosos: contribuições para os serviços de saúde. Revista Latino Americana de Enfermagem, v. 19, n. 5, p. 1-9, 2011. Disponível em: < http://dx.doi.org/10.1590/S0104-11692011000500022>. DOI: 10.1590/S010411692011000500022

PIOLA, F. S.; SERVO, L. M. S.; SÁ, E. B.; PAIVA, A. B. estruturas de financiamento e gasto do sistema público de saúde. IN: FUNDAÇÃO OSWALDO CRUZ. A saúde no Brasil em 2030 - prospecção estratégica do sistema de saúde brasileiro: estrutura do financiamento $e$ do gasto setorial. Rio de Janeiro: Fiocruz/Ipea/Ministério da Saúde/Secretaria de Assuntos Estratégicos da Presidência da República, v. 4, 2013. 168 p.

REIS, L. A.; REIS, L. A.; TORRES, G. V. Impacto das variáveis sociodemográficas e de saúde na capacidade funcional de idosos de baixa renda. Ciência Cuidado Saúde, v. 14, n. 1, p.847-854, jan-mar/2015. Disponível em: < http://dx.doi.org/10.4025/cienccuidsaude.v14i1.19585>. 10.4025/cienccuidsaude.v14i1.19585

RIGON, E.; DALAZEN, J. V. C.; BUSNELLO, G. F.; KOLHS, M.; OLSCHOWSKY, A.; et al. Experiências dos idosos e profissionais da saúde relacionadas ao cuidado pela 
estratégia saúde da família. Revista de enfermagem UERJ, Rio de Janeiro, v. 4, n. 24, e17030, 2016. Disponível em: < http://dx.doi.org/10.12957/reuerj.2016.17030>. DOI:10.12957/reuerj.2016.17030.

SANTOS, G. S; CUNHA I. C. K. O. Avaliação da qualidade de vida de mulheres idosas na comunidade. Revista. Enfermagem Centro Oeste Mineiro. v. 4, n. 2, p. 1135-1145, mai-ago, 2014. Disponível em: < DOI: http://dx.doi.org/10.19175/recom.v0i0.593>. DOI: 10.19175/recom.v0i0.593.

Sistema de Informação da Atenção Básica. Ministério da Saúde. Número de consultas médicas de indivíduos maiores de 60 anos por tipo de equipe segundo modelo de atenção. 2017. Disponível em: http://tabnet.datasus.gov.br/cgi/tabcgi.exe?siab/cnv/SIABSMG.def

SILVA, A. M. M.; MAMBRINI, J. V. M.; PEIXOTO, S. V.; MALTA, D. C.; LIMACOSTA, M. F. Uso de serviços de saúde por idosos brasileiros com e sem limitação funcional. Revista de Saúde Pública, São Paulo, v. 51, p. 1-10, 2017. Disponível em: <https://doi.org/10.1590/S1518-8787.2017051000243> DOI: 10.1590/S15188787.2017051000243

SILVA, C. S. O.; PEREIRA, M. I.; YOSHITOME, A. Y.; RODRIGUES NETO, J. F.; BARBOSA, D. A. Avaliação do uso de medicamentos pela população idosa em Montes Claros, Minas Gerais, Brasil. Escola Anna Nery, v. 14, n. 4, p. 811-18, 2010. Disponível em: <http://dx.doi.org/10.1590/S1414-81452010000400022>. DOI: 10.1590/S1414-81452010000400022.

SIMÕES, C. C. S. Relações entre as alterações históricas na dinâmica demográfica brasileira $e$ os impactos decorrentes do processo de envelhecimento da população. Rio de Janeiro: IBGE, Coordenação de População e Indicadores Sociais, 2016. 119 p.

TAVARES, N. U. L.; COSTA, K. S.; MENGUE, S. S.; VIEIRA, M. L. F. P.; MALTA, D. C.; et al. Uso de medicamentos para tratamento de doenças crônicas não transmissíveis no Brasil: resultados da Pesquisa Nacional de Saúde, 2013. Epidemiologia e Serviços Saúde, Brasília, v. 2, n. 24, p. 315-323, abr-jun/2015. Disponível em: <http://dx.doi.org/10.5123/S1679-49742015000200014>. DOI: 10.5123/S1679-49742015000200014. 